\title{
A novel approach for face detection using hybrid skin color model
}

\author{
Shalini $\operatorname{Yadav}^{1}$ (D) Neeta Nain ${ }^{1}$
}

Received: 31 May 2016 / Accepted: 2 August 2016 / Published online: 13 August 2016

(C) Springer International Publishing Switzerland 2016

\begin{abstract}
Human face detection has become an area of interest in various biometric applications such as crowd surveillance, human-computer interaction, and many security related areas. It is a major field of current research because there is no deterministic algorithm to find the face(s) in a given image. Face detection is challenging due to varying illumination conditions, pose variations, the complexity of noises and image backgrounds. In this paper, a localized approach for face detection based on skin color segmentation and facial features is proposed. Algorithm proficiently analyzes various skin color models such as $R G B, Y C b C r, H S V$ and their combinations for the skin color detection because skin segmentation decreases the computational complexity. The segmented face regions are further classified using a set of facial features such as eye-mouth hole detection, bounding box, and eccentricity ratio. This method is tested on four databases: Bao database contains 157 images, Muct database contains 751 images, FEI database contains 2800 images and GT AV database contains 1188 images. The algorithm achieves an average accuracy of $97.85 \%$. Comparison with Viola Jones, face detection using skin color model, and fast face detection based on skin segmentation and facial features methods has also been done. Further, we apply this method to cartoon characters. Our method works with images with some conditions. First, input images are not monochrome images but color images. Second, the characters skin color has to be near real people skin color. Skin color region is extracted from the image and after this apply the facial fea-
\end{abstract}

Shalini Yadav

yadavshalini45@gmail.com

Neeta Nain

nnain.cse@mnit.ac.in

1 Department of Computer Science and Engineering, Malaviya National Institute of Technology, Jaipur, India tures to locate faces in an image. The proposed approach for cartoon characters faces detection performed well and achieves a good accuracy of $76.00 \%$.

Keywords Image segmentation - Face detection - Color model $\cdot$ Median filter $\cdot$ Euler number $\cdot$ Eccentricity

\section{Introduction}

With the concern of security system and media, more efficient and useful techniques for human-computer interaction (HCI) are being filtered which do not depend on traditional sources such as keyboards, mouse, and displays. The fast growing research in face processing is based on the fact that images can be used for extracting information about a user's identity, state and according to state, computers can then react. Detection of human faces is a major concern in image processing field and various applications, such as time tracking service, outdoor surveillance camera service, smart captcha, secured access, video chat service.

We believe that face detection and recognition for cartoon characters are also important research topics because various applications using them can be contemplated and cartoon characters are world-widely spreading.

There are many applications of face detection of cartoon characters. Character search is one of the most important applications. An image containing a cartoon character is given as an input, and it is possible to automatically search images containing the same cartoon character from Web or database. This function is currently not available in existing search systems (for example, Google Image Search).

What is face detection? "Face detection" as the keyword itself reveals its meaning that it is a method of searching faces in an image, and if present, then returns the image location and 
vicinity of each face. The challenges with face detection can be described to the following factors:

1. Pose Due to the position of camera-face (frontal, $45^{\circ}$, profile, upside down), face in the images vary and some facial features such as an eye or the nose may become partially or completely hide.

2. Presence or absence of structural components Facial features such as beards, eye glasses may or may not be present, and there are variations among these components including shape, color, and size.

3. Facial expression Facial expressions such as sad, happy, angry, may change the appearance of face.

4. Occlusion Faces may be partially tilled by other components. Due to more than one faces in an image, faces may partially occlude with each other.

5. Image orientation According to camera's optical axis, face images directly vary for different rotations.

6. Imaging conditions While forming an image, factors such as lighting condition and camera characteristics affect the appearance of a face.

To handle these difficulties researchers proposed different methods [1]. Face detection techniques are broadly distributed into traditional and enhanced approaches. The error rate is high in traditional approaches because they are affected by the lighting environment and profile orientations. These techniques are working on Haar features, or templates which are not efficient for nowadays. In addition there are less rules in traditional approaches which sometimes increase false rate in detection. Enhanced approaches perform better than traditional techniques because it handles the situation in adverse conditions, e.g., occlusion, illumination changes, etc. These techniques give detailed description of every feature and make strong rules for detection. Enhanced techniques use some advances in face detection approach, e.g., neural networks, skin color segmentation, etc. Various enhanced techniques are proposed for face detection, i.e., the S-AdaBoost algorithm [2], skin color-based algorithm $[3,4]$, neural networks [5,6], Bayes classifier [7], chrominance based [8].

In this paper, we present a technique for face detection which is based on skin color segmentation using various color models such as $R G B, Y C b C r$ and $H S V$, and also combination with these color models. In an image, the face is foreground region and rest is background region. Differentiating skin region and the non-skin region in the image is a major step in face detection algorithm since it reduces the time complexity of the algorithm. Here, we analyze that hybrid skin color model gives a better result than the single skin color model. This paper detects faces in an image using the combination of the skin segmentation and facial features. It has been found that using skin color segmentation, the accuracy of the algorithm is improved and gives the better result than other approaches. This method also works for cartoon faces where cartoon skin color should be near to human skin color. The method is tested on a large number of cartoon characters and achieves good accuracy.

The paper is organized in the following manner: section 2 describes the related work. Section 3 describes the proposed methodology. Section 4 gives experimental results. Section 5 summarizes the conclusions.

\section{Related work}

Face detection methods are broadly classified into four areas: template matching methods, feature invariant methods, knowledge-based methods and appearance-based methods [9].

1. Template matching methods In this method, it stores several standard patterns to describe the face either as a whole or as a set of facial features independently [10-12]. It finds the location of faces based on correlation values with a standard face pattern. The approach faces some difficulties like hard to represent standard templates fit for different: poses, orientations, facial expression, illumination conditions, etc.

2. Feature invariant methods It uses facial features, such as eyes, nose, ear, lip and skin color [13-18] for face detection. These methods could handle varying lighting conditions, out of the plane rotation or in-plane rotations. 3. Knowledge-based methods These methods are based on rules $[19,20]$ that describe the relationships between facial features. These methods are mainly designed for localization of faces. But the accuracy and efficiency of these methods are greatly affected by rules designed for face detection. If high constraint rules apply, then it gives low detection rate while easy rules give false detection rate.

4. Appearance-based methods These methods need to be trained on some set of images. After this, test images are applied on this trained model for face detection [21-25]. There is no need of predefined templates. Neural network based face detection technique comes under this method.

\section{Proposed methodology}

In this paper, hybrid approach is used because we combine feature invariant and knowledge-based approach. Here, feature invariant consist skin color segmentation and knowledgebased approach consist Euler computation, bounding box and eccentricity ratio. The model takes advantage of the additional hue and chrominance information of the image on 
RGB properties to raise the segregate between skin pixels and non-skin pixels. In our approach, skin regions are segmented based on the $R G B$ boundary rules and also additional new rules for the $H S V$ and $Y C b C r$ color spaces.

Figure 1 gives the overview of the proposed face detection algorithm, which consists two stages: training stage and detection stage. Skin color range is determined using some set of images from the database. Three popular color spaces $R G B, H S V$ and $Y C b C r$ are used to develop the hybrid skin color model for skin segmentation. According to skin color distributions, boundary rules for each color model are constructed.

In the first step of the detection stage, we applied the image enhancement process to minimize illumination varying effect. Then skin regions are segmented based on the $R G B$ boundary rules and also additional new rules for the $H S V$ and $Y C b C r$ color spaces. After that, combinations of facial features are applied to the extracted skin regions to eliminate possible non-face skin regions. Finally, the last step marks all the face regions in the image and returns them as detected faces.

\subsection{Image enhancement}

Skin color segmentation is dependent on light variations. Skin color segmentation is affected by the lighting condition of image. To increase the accuracy of skin tone segmentation, we perform image enhancement process on images [26]. We enhance the important details of an image by adjusting the light contrast of the image. Image enhancement process has been done because if the light is too dark or too bright, skin segmentation would not perform well and give false result or no result. For this purpose, we use $\mathrm{YCbCr}$ color model (Fig. 2). The following steps summarize image enhancement process:

1. Convert $R G B$ image into $\mathrm{YCbCr}$ image and normalize the $Y$ component.

2. Calculate the average value of $Y$.

$Y \operatorname{avg}=\frac{\Sigma Y(x, y)}{W * H}$

where $W$ and $H$ are the width and height of the image, respectively. We set the threshold value $T$ according to $Y$ avg value. We analyze the different images with different light sources from dark to bright and set the threshold values for $Y$ avg and $T$. Initial value of $T$ is 1 which means if lighting of image is good, then pixel values do not change.

$T= \begin{cases}1.4 & \text { Yavg }<64 \\ 0.6 & \text { Yavg }>192\end{cases}$
3. Modify $R$ (red) and $G$ (green) component of the image using threshold values, and combine Rnew, Gnew and B (blue) to get the final color image.

$$
\begin{aligned}
& \text { Rnew }= \begin{cases}R^{T} & T \neq 1 \\
R & \text { otherwise }\end{cases} \\
& \text { Gnew }= \begin{cases}G^{T} & T \neq 1 \\
G & \text { otherwise }\end{cases}
\end{aligned}
$$

\subsection{Skin region segmentation}

Then skin regions are segmented based on the RGB boundary rules and also additional new rules for the $\mathrm{HSV}$ and $\mathrm{Y} \mathrm{CbCr}$ color spaces. There are two steps for skin segmentation: first, prepare skin color model using training image, and second, apply the bounding rules on test images. These steps are as follows:

\subsubsection{Preparation of skin color models}

To build the skin color bounding rules for color spaces, a set of training images were used to analyze the skin color distribution range in various color subspace. Our training set consists 60 color images which are obtained from the various sources such as the internet which covers a wide range of skin color variations and ethnicity. This training set contains images which were captured in normal uniform illumination, daylight or flash light illumination.

\subsubsection{Skin color bounding rules}

The training images were analyzed in the $R G B, H S V$ and $Y C b C r$ spaces [27]. Images that are represented by $R G B$ color space are less efficient to construct skin color models because skin color region is not well-distinguished in all three channels intensities [28,29]. Boundary values are obtained from the training images:

\section{RGB}

$V=[R, G, B]$

$(R>95 \cap G>40 \cap B>20 \cap(\max (V)-\min (V)>$ $15) \cap(\operatorname{abs}(R-G) \geq 15) \cap R>G \cap R>B)$

\section{$\mathrm{YCbCr}$}

$(85 \leq C b \leq 135) \cap(10 \leq C r \leq 45) \cap(Y \geq 80)$

\section{HSV}

$(H>0 \cap H<35) \cup(H>325 \cap H<360) \cap(S>$ $0.2 \cap S<0.6) \cap(V \geq 20)$

Our segmentation technique, which uses all three color models, was developed to raise the face detection accuracy, as will be examined in the experimental results. Results of image segmentation using various color models are shown in Fig. 3, 


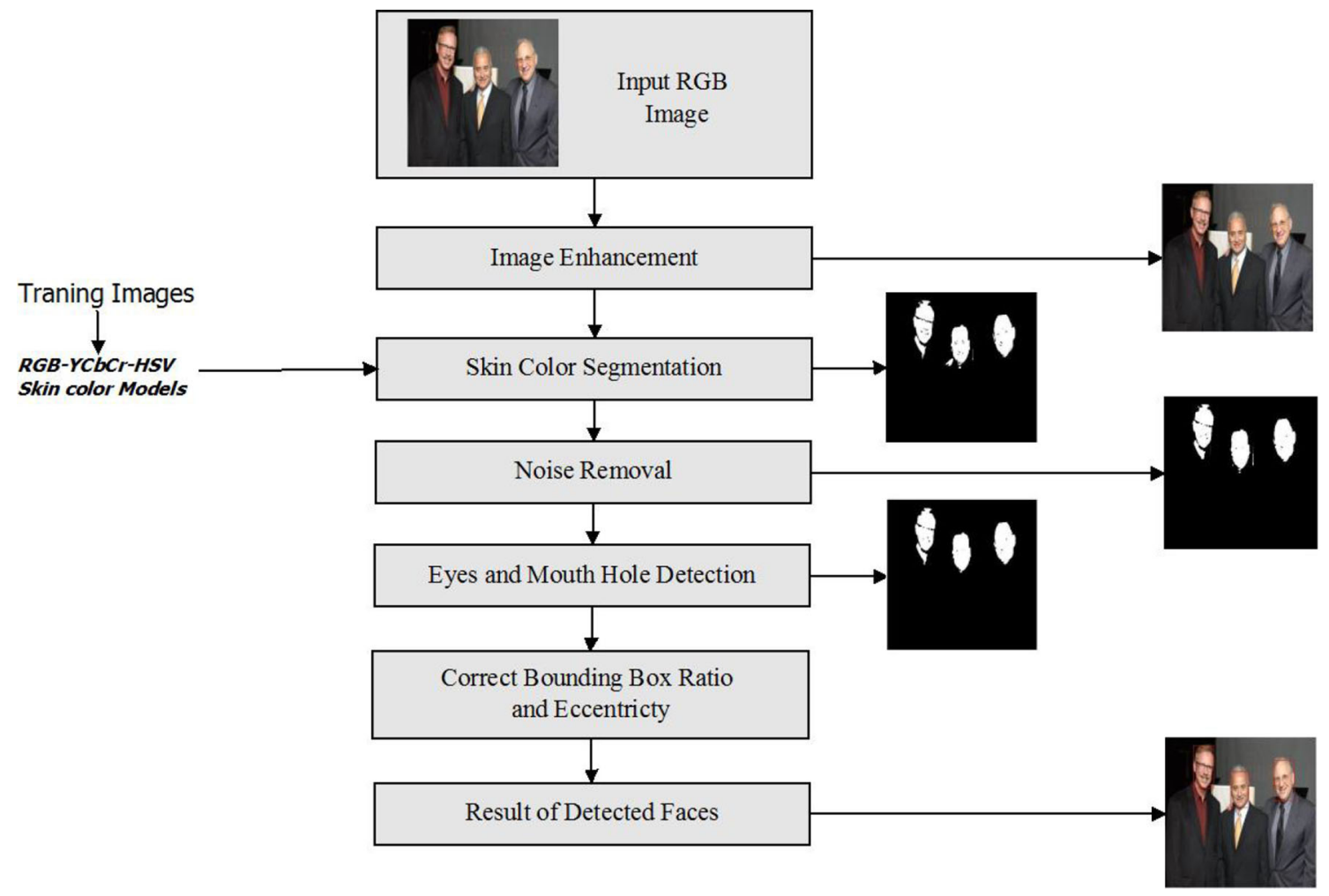

Fig. 1 Framework of the proposed face detection algorithm

Fig. 2 a Input image and b enhanced image

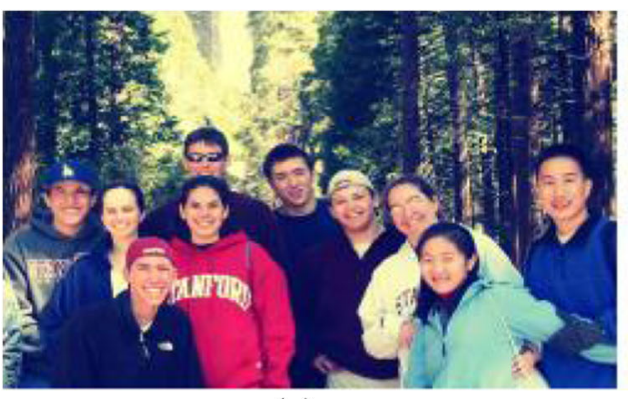

(a)

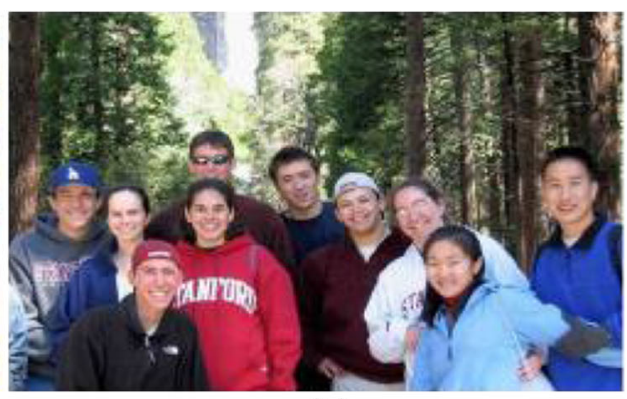

(b) where pixels with near to skin color becomes white while others are black.

\subsection{Noise removal}

Noise is the result of errors while processing an image which results in pixel values that do not reflect the true intensities of the real scene. Noise can be introduced into an image due to some reasons such as:

- While scanning an image from a photograph made on film, the film grain is a source of a noise. If a film is damaged, then it results in noise.

- If the image is taken directly in a digital format, the mechanism for collecting the data can introduce noise.
- Electronic transmission of image data can introduce noise.

For better performance, there is a need to filter the noise. Different types of noise filter are available such as low pass filter, FFT, and median filter. We use median smoothing filter on image to smoothen its noise. For noise removal, one can use either low pass filter or FFT but the problem with these filters is that sometimes they also remove important information. Median filter perform better than these filters. The process followed by median filtering is:

- It is similar to an averaging filter, in that each output pixel is set to an average of the pixel values in the neighborhood of the corresponding input pixel. 


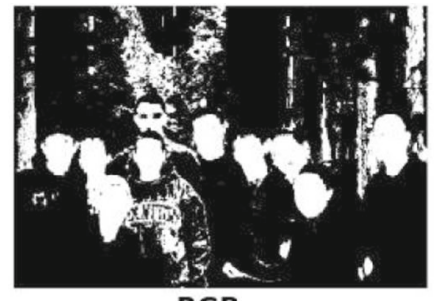

RGB

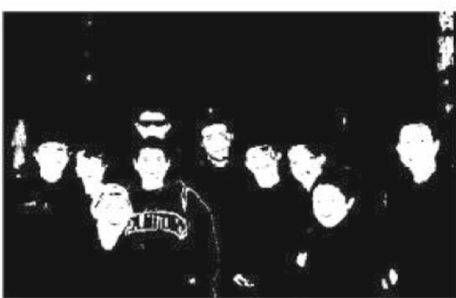

$\mathrm{RGB}$ and $\mathrm{YCbCr}$

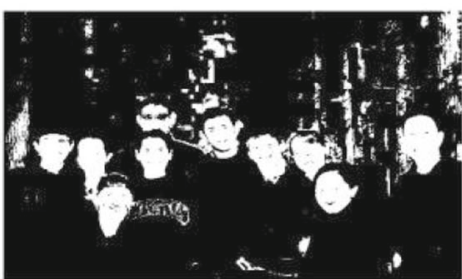

$\mathrm{YCbCr}$

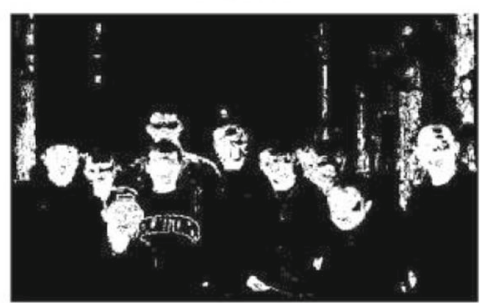

RGB and HSV

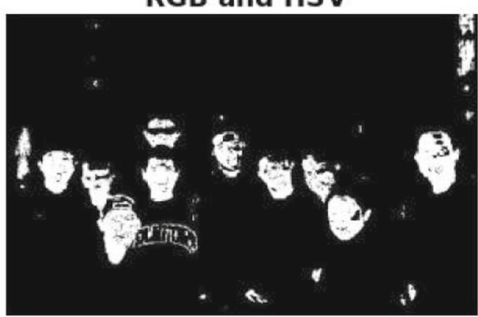

RGB and YCbCr and HSV

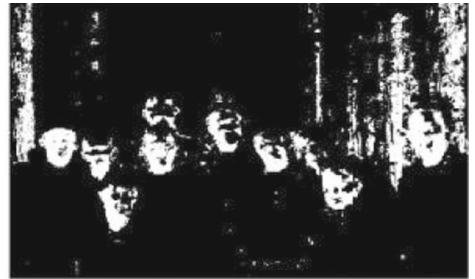

HSV

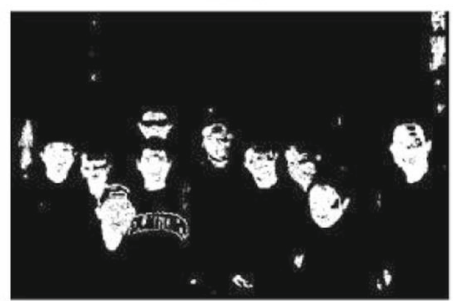

HSV and YCbCr

Fig. 3 Results of skin color segmentation with $R G B, Y C b C r$, and $H S V$ color models

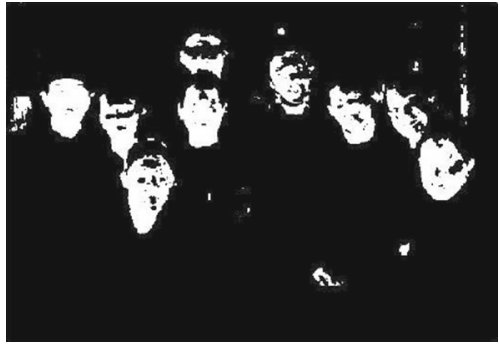

Fig. 4 Noise removal result on skin segmented image

- The value of an output pixel is determined by the median of the neighborhood pixels, rather than the mean.

- The median is much less sensitive than the mean to extreme values (called outliers).

Median filtering is therefore better able to remove these outliers without reducing the sharpness of the image. Result of noise removal is shown in Fig. 4.

\subsection{Euler number calculation}

Since faces contain holes due to eyes and mouth so by calculating the number of holes, we discard the region that does not contain any holes. To perform Euler number computation, we consider each skin region as individual and process the

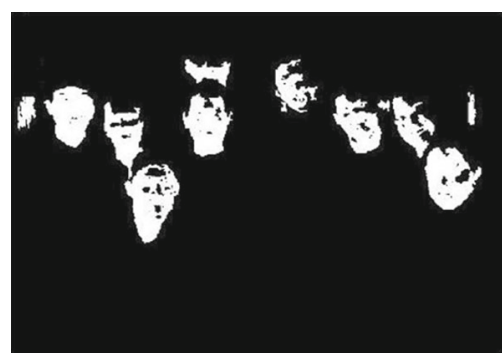

Fig. 5 Euler computation result on skin segmented image

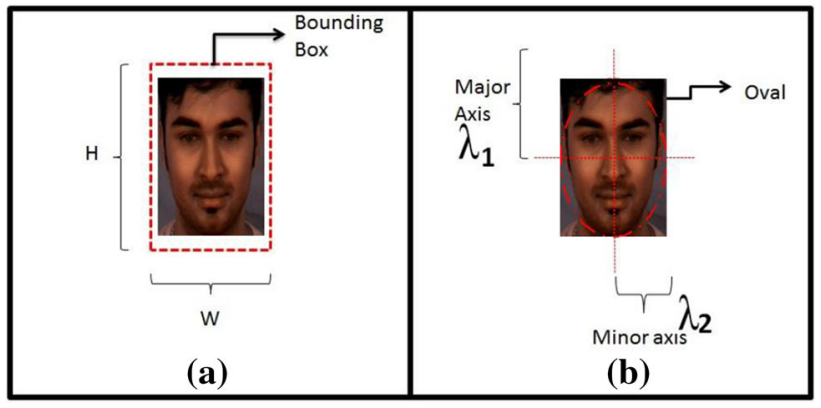

Fig. 6 a Bounding box and b eccentricity

Euler number computation. For this purpose, Euler formula is used:

$E=C-H$ 

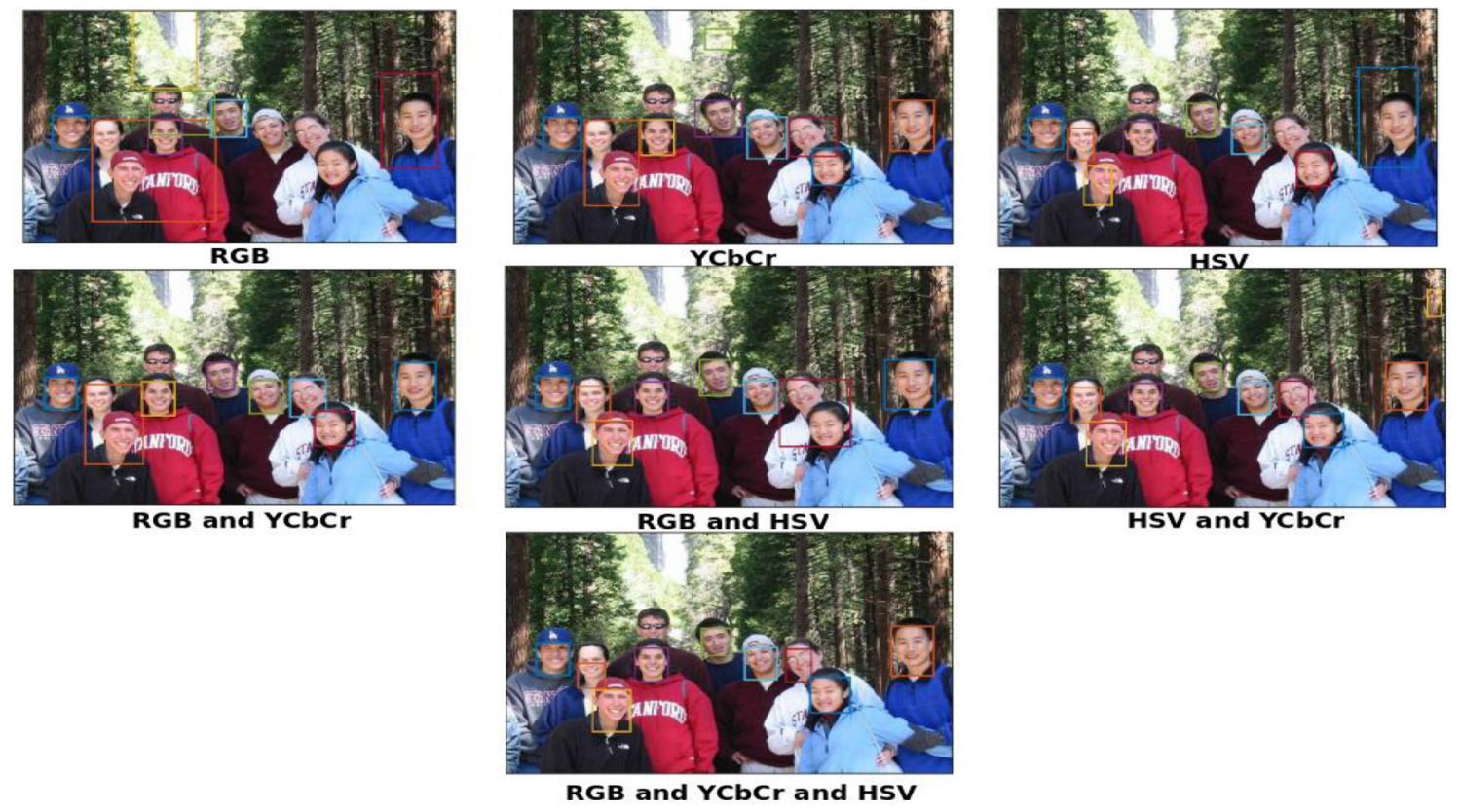

RGB and YCbCr

HSV and YCbCr

Fig. 7 Face detection results with different skin color models

Where $E$ is the Euler number, $C$ is the number of connected components, and $H$ is the number of holes. The result of Euler computation is shown in Fig. 16.

\subsection{Bounding box ratio and eccentricity}

For further verification of face, we process the image with bounding box ratio and eccentricity to determine the likelihood of a skin region being a face region. Both conditions are as shown in Fig. 6.

The height of the bounding box for face is greater than the width. Threshold values for bounding box is decided by analyzing width to height ratio of 100 human faces from internet and databases. We compute the ratio of width $W$ to height $H$ of bounding box $B_{i}$ :

$R=\frac{W}{H}$
$B_{i}= \begin{cases}\text { true } & \text { if } R>0.35 \\ \text { false } & \text { otherwise }\end{cases}$

Since human face is an oval type, so here eccentricity property measures the ratio of the minor axis to major axis of a bounding ellipse.

$E c_{i}= \begin{cases}\text { false } & \text { if } E_{i}>0.89 \text { or } E_{i}<0.1 \\ \text { true } & \text { otherwise }\end{cases}$

Here $E_{i}$ is the eccentricity value. After calculating $B_{i}$ and $E c_{i}$, we apply $A N D\left(B_{i} A N D E c_{i}\right)$ constraint on region. If condition is true then it is marked as face region, otherwise discarded. Figure 7 shows the face detection result with various color models. 
The complete approach is illustrated in Algorithm 1:

Algorithm 1 Face Detection Algorithm

Begin

1. Read an image.

2. Convert $R G B$ image into $Y C b C r$ image.

3. For lightning compensation perform following steps:

a Find the minimum $\min (Y)$ and maximum $\max (Y)$ value of $Y$ component and normalize the $Y$ component.

b Calculate the average value of $Y$.

$$
Y a v g=\frac{\Sigma Y(x, y)}{W * H}
$$

c Set threshold value with the following conditions:

$$
T= \begin{cases}1.4 & \text { Yavg }<64 \\ 0.6 & \text { Yavg }>192\end{cases}
$$

d Modify the $R($ red) and $G($ green) component of image using threshold

$$
\text { Rnew }=\left\{\begin{array}{ll}
R^{T} & \mathrm{~T} \neq 1 \\
R & \text { otherwise }
\end{array}, \quad \text { Gnew }= \begin{cases}G^{T} & \mathrm{~T} \neq 1 \\
G & \text { otherwise }\end{cases}\right.
$$

e Combine Rnew, Gnew and B(blue) to get the final color image.

4. Perform skin color segmentation using following threshold value:

if $V=[R, G, B]$;

$(R>95 \cap G>40 \cap B>20 \cap(\max (V)-\min (V)>15) \cap(\operatorname{abs}(R-G) \geq 15) \cap R>G \cap R>B)$

AND

$Y \geq 80 \cap 10 \leq C r \leq 45 \cap 85 \leq C b \leq 135$

AND

$(H>0 \cap H<35) \cup(H>325 \cap H<360) \cap(S>0.2 \cap S<0.6) \cap(V \geq 20)$

else

Skin region

Non skin region

5. Perform noise removal using median filter.

6. Calculate the number of connected component in skin segmented image.

7. Perform euler computation E.

if $E \geq 0$

else

No hole, discarded the region.

Accepted.

8. Put the bounding box ratio $B_{i}$ and eccentricity ratio $E c_{i}$ on image.

9. if $B_{i} \cap E c_{i}=$ true

Facial region

else

Non-facial region.

End 


\section{Experimental results}

\subsection{Human face detection}

The face detection system was implemented using MATLAB on a $2.4 \mathrm{GHz}$ Intel Core i3 machine running on $3 \mathrm{~GB}$ RAM. Databases which are used for testing are Bao database, Muct Landmark database [33], FEI database [34], and GTAV database [35]. Muct database contains single face images of a person with different orientations, Bao database contains pictures with more than one faces, FEI database contains 14 images for each of 200 individuals, a total of 2800 images with a white homogenous background in an upright frontal position with profile rotation of up to about $180^{\circ}$, occlusion (glasses and beard) and facial expression, and $G T A V$ database includes a total of 44 persons with 27 pictures per person which correspond to different pose views under three different illuminations (environment or natural light, strong light source from an angle of $45^{\circ}$, and finally an almost frontal mid-strong light source). Figures 12, 13, and 14 show the face detection result on these datasets. To evaluate our experiments, we defined two performance metrics: detection success count (DSC) and false detection count (FDC). DSC is defined as the number of correctly detected faces over the actual number of faces in the image.

$\mathrm{DSC}=\frac{\mathrm{TP}}{\mathrm{TP}+\mathrm{FN}} \times 100$

FDC is defined as the number of false detections over the total number of detections.

$\mathrm{FDC}=\frac{\mathrm{FP}}{\mathrm{FP}+\mathrm{TN}} \times 100$

A confusion matrix as shown in Table 1 is the standard metrics used for the evaluation of face detection.

Table shows the comparison between our approach, Viola Jones approach [30], Anil K. Jain approach [31] and fast face detection based on skin segmentation and facial features [32]. Tables 2, 3, 4, 5 show that our approach increases the accuracy of detection. The algorithm is more efficient because the time complexity of algorithm is $O\left(n^{2}\right)$ where $n$ is the no. of

Table 1 Metrics for evaluation of face detection

\begin{tabular}{llll}
\hline Confusion matrix & & Predicted label & \\
\cline { 3 - 4 } & & Non-faces & Faces \\
\hline Actual & Non-faces & True & False \\
label & negative & positive \\
& & $(\mathrm{TN})$ & $($ FP) \\
& & False & True \\
& Faces & negative & positive \\
& & $($ FN) & $(\mathrm{TP})$ \\
\hline
\end{tabular}

Table 2 Comparative analysis of frontal faces with different lighting condition on Muct database [33]

\begin{tabular}{llll}
\hline Approach & Images & FDC (\%) & DSC (\%) \\
\hline $\begin{array}{l}\text { Viola } \\
\text { Jones [30] }\end{array}$ & 751 & 6.7 & 95.87 \\
$\begin{array}{l}\text { Face } \\
\text { detection in } \\
\text { color } \\
\text { images [31] }\end{array}$ & 751 & 5.9 & 99.20 \\
$\begin{array}{l}\text { Fast face } \\
\text { detection }\end{array}$ & 751 & & \\
$\begin{array}{l}\text { based on } \\
\text { skin seg- } \\
\text { mentation } \\
\text { and facial }\end{array}$ & & 4.5 & 99.20 \\
$\begin{array}{l}\text { fea- } \\
\text { tures [32] }\end{array}$ & & & \\
$\begin{array}{l}\text { Proposed } \\
\text { method }\end{array}$ & 751 & & \\
\hline
\end{tabular}

Table 3 Comparative analysis of multiple faces in image with different orientation condition on Bao database [33]

\begin{tabular}{llll}
\hline Approach & Images & FDC (\%) & DSC (\%) \\
\hline $\begin{array}{l}\text { Viola } \\
\text { Jones [30] }\end{array}$ & 157 & 9.5 & 82.80 \\
$\begin{array}{l}\text { Face } \\
\text { detection in } \\
\text { color }\end{array}$ & 157 & 8.1 & 89.17 \\
images [31] & & & \\
$\begin{array}{l}\text { Fast face } \\
\text { detection } \\
\text { based on } \\
\text { skin seg- } \\
\text { mentation } \\
\text { and facial }\end{array}$ & 157 & 6.7 & 94.26 \\
fea- \\
tures [32]
\end{tabular}

components in the image. Figures $8,9,10$, and 11 show the performance graph of our method with different methods.

\subsection{Cartoon face detection}

We also tested our method on cartoon character face detection with slight modification. Since anime-skins are not equivalent to human skin color [20]. They are created by artists, and their color varies based on age, race, gender, etc. To build the skin color model, a set of images were used to analyze the properties and distribution of skin color in various color subspaces. Our image set is composed of 70 color images obtained from the various sources such as the internet which covers a wide range of skin color variations and ethnicity. 
Table 4 Comparative analysis of profile faces on FEI database [34]

\begin{tabular}{llll}
\hline Approach & Images & FDC (\%) & DSC (\%) \\
\hline $\begin{array}{l}\text { Viola } \\
\text { Jones [30] }\end{array}$ & 2800 & 10.7 & 95.87 \\
$\begin{array}{l}\text { Face } \\
\text { detection in } \\
\text { color }\end{array}$ & 2800 & 15.9 & 68.95 \\
images [31] & & & \\
$\begin{array}{l}\text { Fast face } \\
\text { detection }\end{array}$ & 2800 & 8.5 & \\
based on \\
skin seg- \\
mentation \\
and facial \\
$\begin{array}{l}\text { fea- } \\
\text { tures [32] }\end{array}$
\end{tabular}

Table 5 Comparative analysis of profile faces on GTAV database [35]

\begin{tabular}{llll}
\hline Approach & Images & FDC (\%) & DSC (\%) \\
\hline $\begin{array}{l}\text { Viola } \\
\text { Jones [30] }\end{array}$ & 1188 & 12.3 & 95.25 \\
$\begin{array}{l}\text { Face } \\
\text { detection in }\end{array}$ & 1188 & 17.8 & 65.7 \\
$\begin{array}{l}\text { color } \\
\text { images [31] }\end{array}$ & & & \\
$\begin{array}{l}\text { Fast face } \\
\text { detection }\end{array}$ & 1188 & 4.2 & \\
$\begin{array}{l}\text { based on } \\
\text { skin seg- } \\
\text { mentation } \\
\text { and facial } \\
\text { fea- } \\
\text { tures [32] }\end{array}$ & & & \\
$\begin{array}{l}\text { Proposed } \\
\text { method }\end{array}$ & & & \\
\hline
\end{tabular}

For skin detection here, we use two color model: $R G B$ and $H S V$. A pixel is a skin if:

\section{RGB}

$120<R<255 \cap 90<G<250 \cap 70<B<218 \cap R>$ $G \cap G>B$

\section{HSV}

$0^{\circ}<H<45^{\circ} \cap V>75 \%$

Applying these threshold values, skin regions are extracted from the given input image. Result of skin segmentation is shown in Fig. 15.

After this Euler number computation $E$ as shown in Eq. 5 is applied on skin extracted region to obtain probable face region as shown in Fig. 5. Skin color component which do not contain any holes are discarded by Euler number computation. To locate faces in an image, apply the bounding box

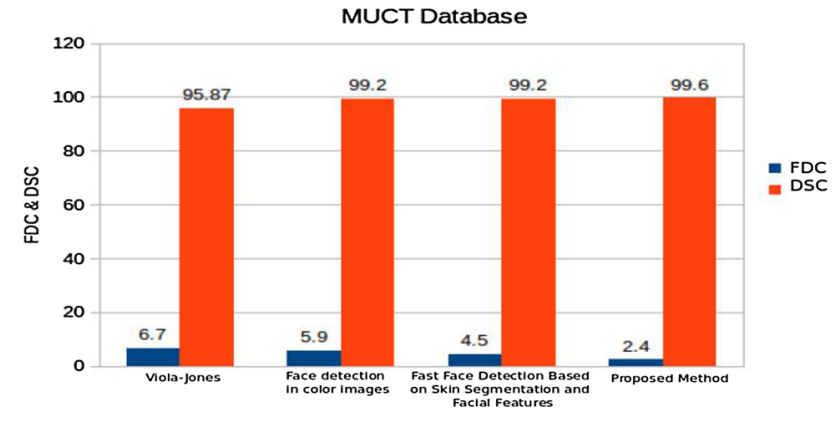

Fig. 8 Performance graph for MUCT Database

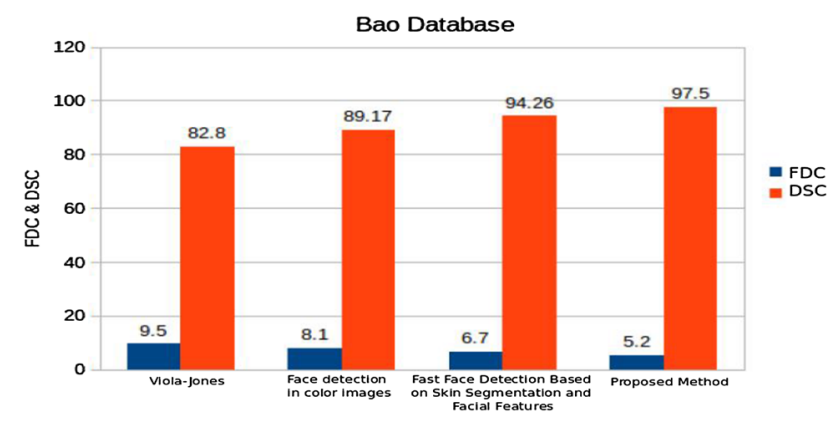

Fig. 9 Performance graph for Bao Database

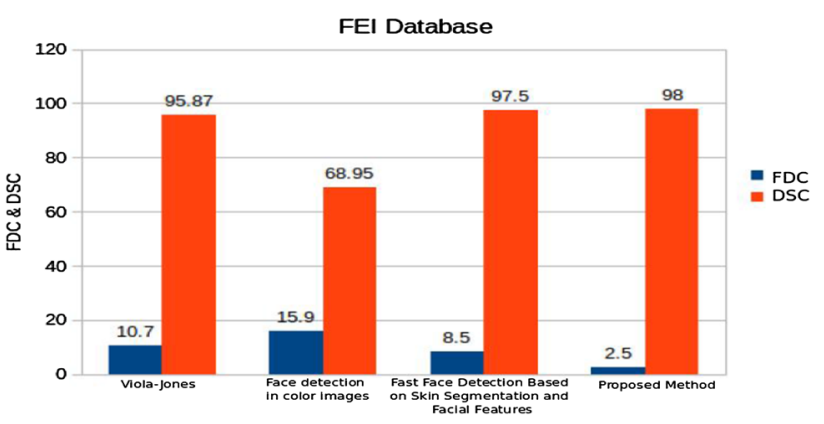

Fig. 10 Performance graph for FEI Database

threshold value. These threshold values are computed by analyzing facial structure of cartoon faces from the dataset. Face detection results is shown in Fig. 17.

$B_{i}= \begin{cases}\text { true } & \text { if } R>0.35 \text { and } R<1.5 \\ \text { false } & \text { otherwise }\end{cases}$

For testing our approach we collected 500 images with 1022 faces from the internet. This image dataset contains the collection of cartoon faces of popular serials and comics. Here, we divide these images into groups according to same group cartoon characters or same serials with different backgrounds. Here, two measures are used for testing our approach, i.e., TPR (true positive rate) and FPR (false 


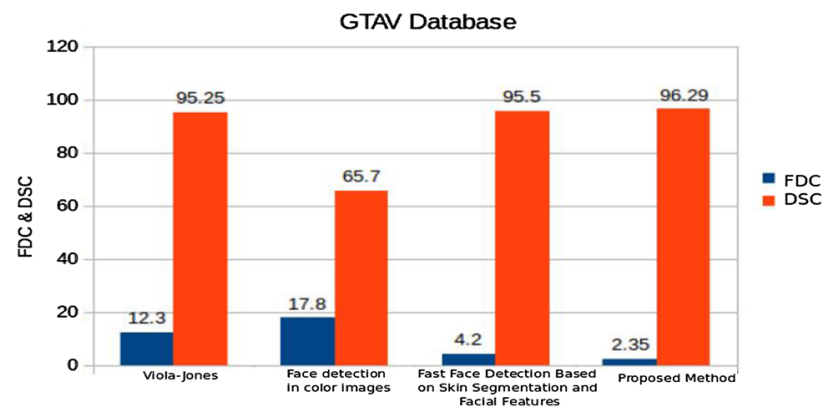

Fig. 11 Performance graph for GTAV Database positive rate):

$\mathrm{TPR}=\frac{\mathrm{TP}}{\mathrm{TP}+\mathrm{FN}}$

$\mathrm{FPR}=\frac{\mathrm{FP}}{\mathrm{FP}+\mathrm{TN}}$

We break these 500 images into five parts; each part contains 100 images which include the same type of characters with different background and poses. We calculate TPR and FPR for each part and make ROC space for every database.

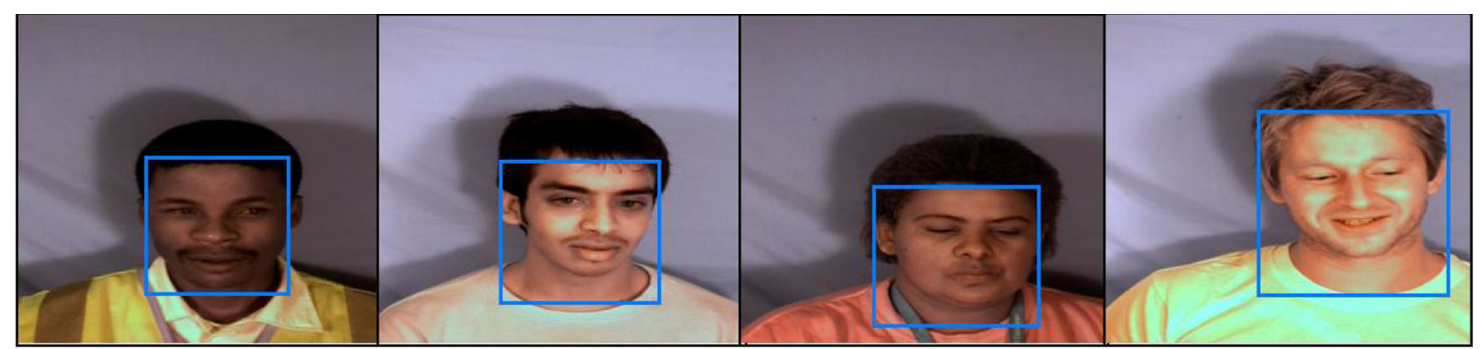

(a)

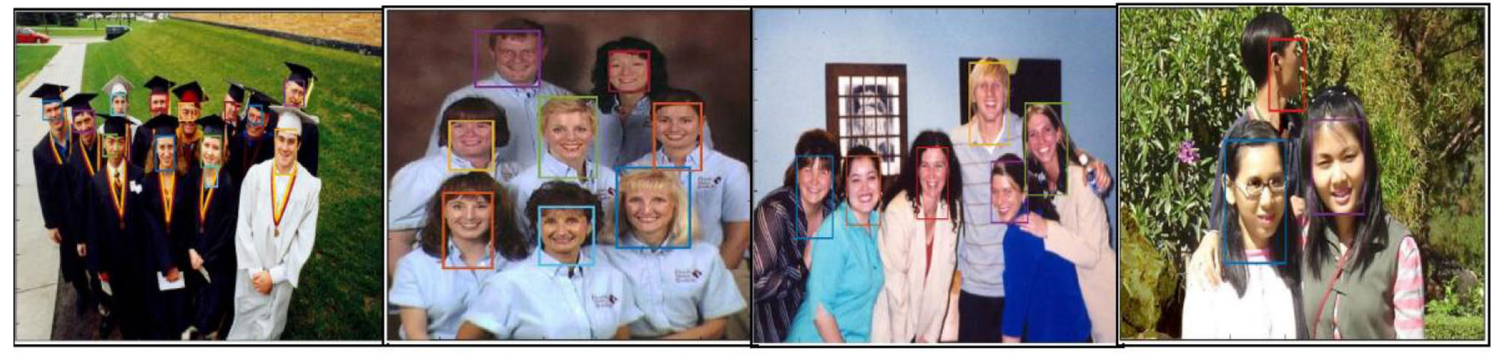

(b)

Fig. 12 Face detection result. a Muct database and $\mathbf{b}$ Bao database
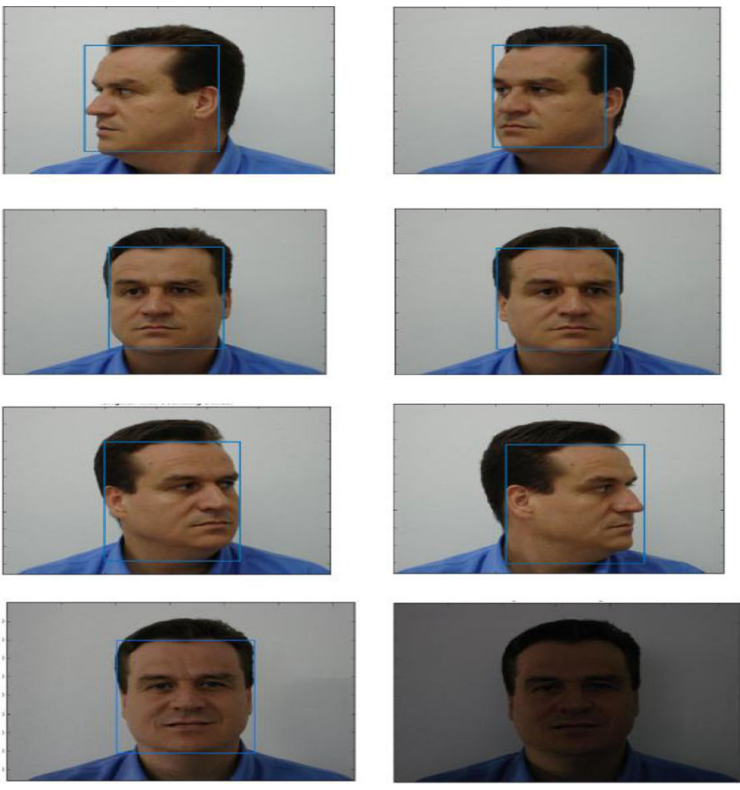
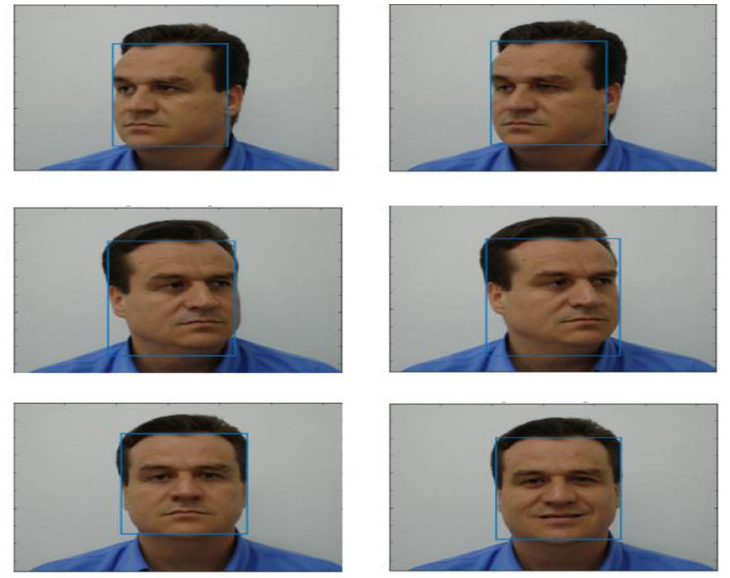

Fig. 13 Face detection result on FEI Database 

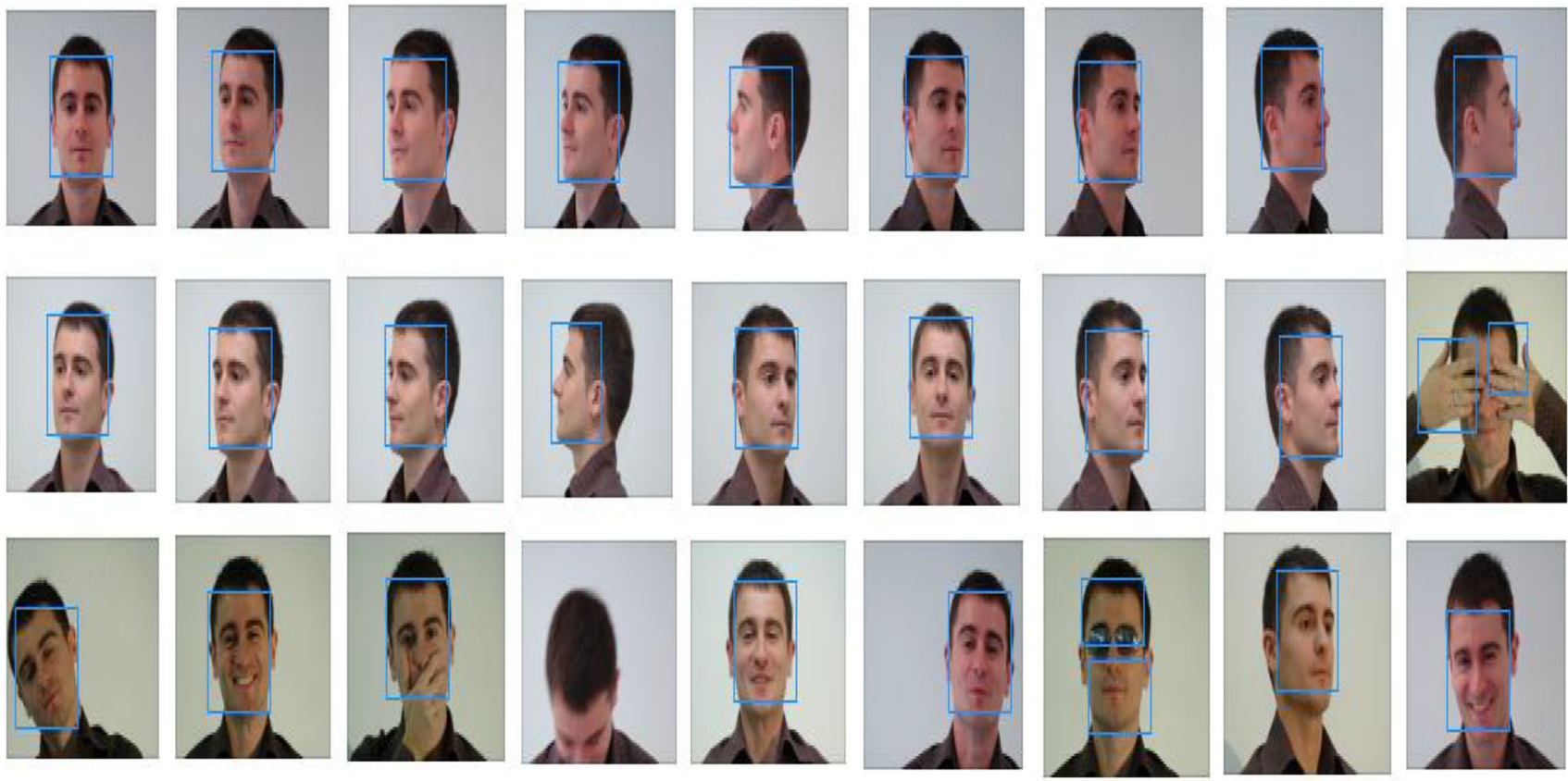

Fig. 14 Face detection result on GTAV Database
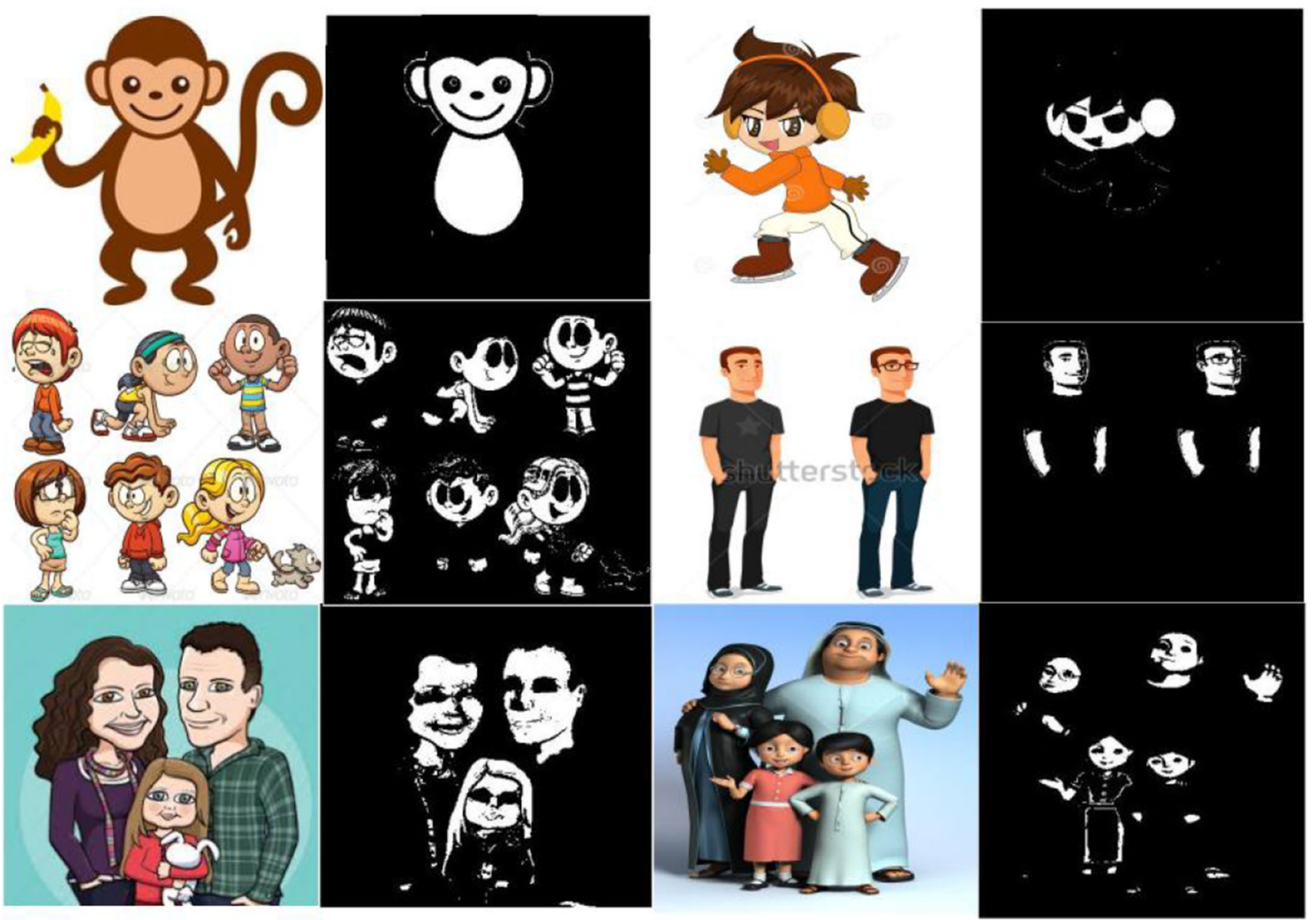

Fig. 15 Skin segmentation result for cartoon characters

Proposed method fails when the background color is similar to skin color as shown in ROC space Fig. 18. ROC curve for proposed method is shown in Fig. 19. Our method achieves an average accuracy of $76.00 \%$ but the performance of this method is decreased when the background color matches to foreground color. 
Fig. 16 Euler computation result for cartoon characters

Fig. 17 Face detection result for cartoon characters
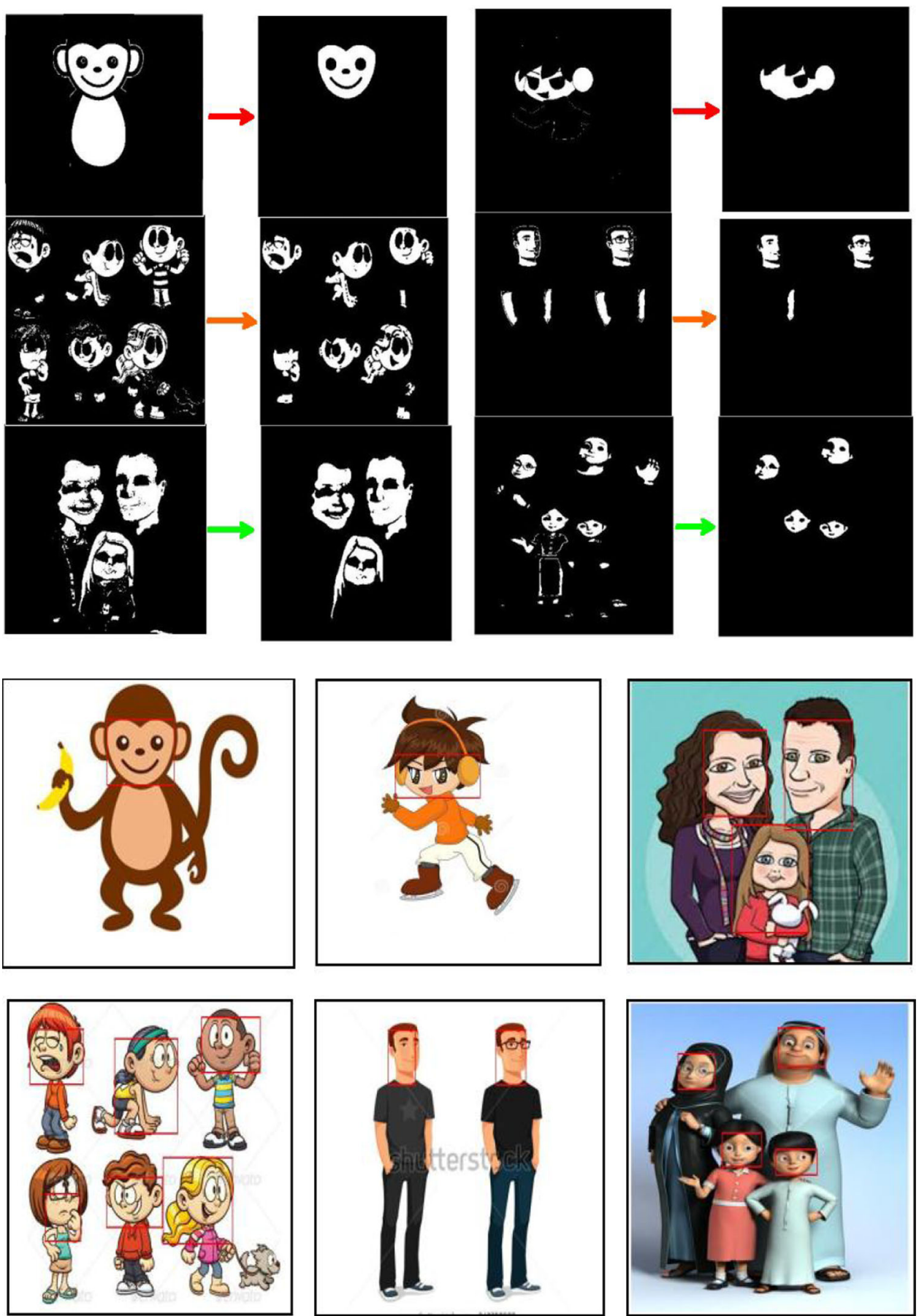

Detection results on three databases: Bao database [33], Muct database [33], FEI database [34], GTAV database [35] are presented where the proposed algorithm achieves an average accuracy of $97.85 \%$, compared to Viola zones [30] with an average accuracy of $92.45 \%$, face detection using skin color model method [31] with an average accuracy of $80.75 \%$, and fast face detection based on skin segmentation and facial features [32] $96.61 \%$. 


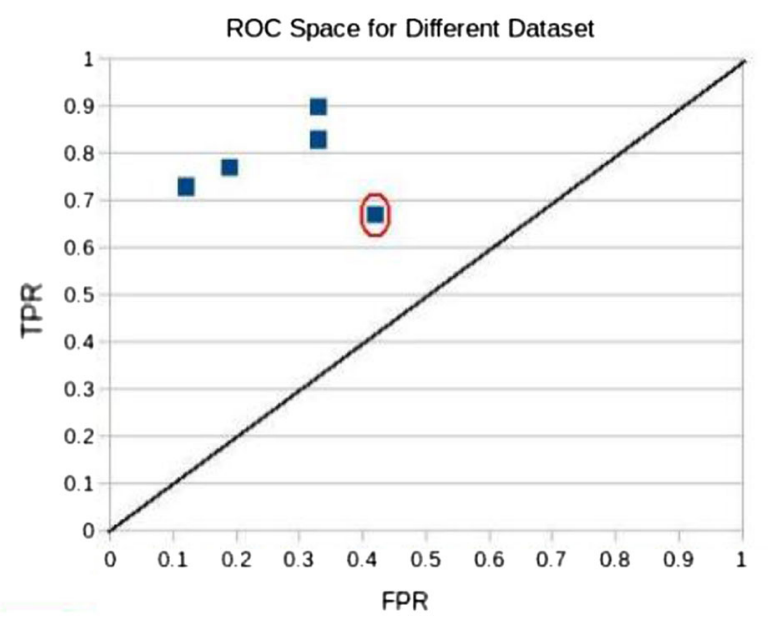

Background color component are skin color type

Fig. 18 Roc space for cartoon face detection

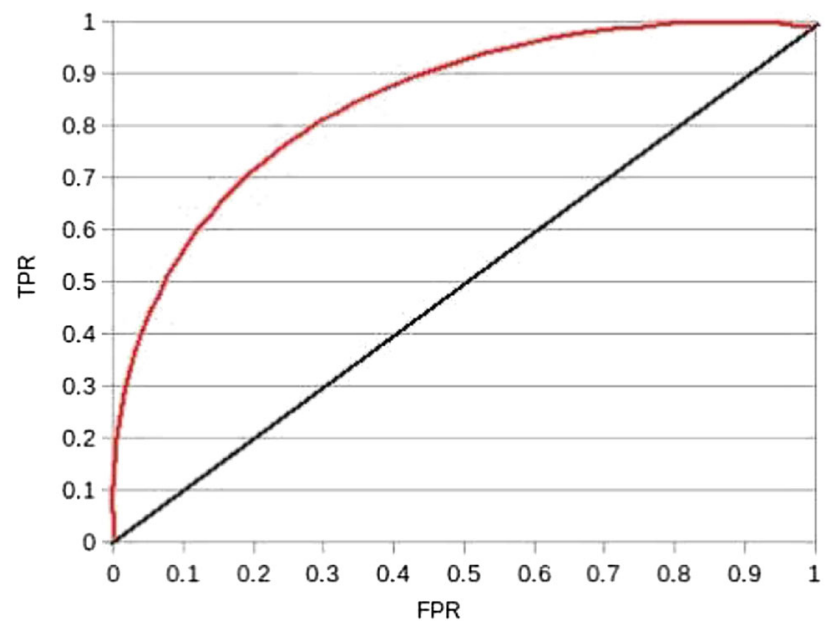

Fig. 19 Roc curve for cartoon face detection

We also test our method on cartoon face detection. The skin region segmentation has done using the combination of RGB, and HSV subspaces, which discriminated skin and non-skin regions. The experimental results showed that our new approach in modeling skin color was able to achieve a good true positive rate (TPR) for various cartoon characters. Our method achieves an average accuracy of $76.00 \%$, but the performance of this method decreases when background color matches to foreground color.

\section{References}

1. Mostafa L, Abdelazeem S (2005) Face detection based on skin color using neural networks. GVIP 05 Conference 7:19-21

2. Sirovich L, Kirby M (1987) Low-dimensional procedure for the characterization of human faces. JOSA A 4(3):519-524
3. Qiang-rong J, Hua-Lan L (2010) Robust human face detection in complicated color images. In: Information Management and Engineering (ICIME), 2010 The 2nd IEEE International Conference IEEE, pp 218-221

4. Vincenzo R, Lisa U (2007) An improvement of adaboost for facedetection with motion and color information. In: Image Analysis and Processing, ICIAP 2007 14th International Conference IEEE, pp 518-523

5. Jain AK, Ross A, Prabhakar S (2004) An introduction to biometric recognition. Circuits Syst Video Technol IEEE Trans 14(1):4-20

6. Shih P, Liu C (2004) Face detection using discriminating feature analysis and support vector machine in video. In: Pattern recognition, ICPR 2004. Proceedings of the 17th International Conference, IEEE, vol 2, pp 407-410

7. Osuna E, Freund R, Girosi F (1997) Training support vector machines: an application to face detection. In: Computer vision and pattern recognition. Proceedings 1997 IEEE Computer Society Conference, IEEE, pp 130-136

8. Chandrappa D, Ravishankar M, RameshBabe D (2010) Automated detection and recognition of face in a crowded scene. Int J Comput Netw Secur 2(6):65-70

9. Zhao W, Chellappa R, Phillips PJ, Rosenfeld A (2003) Face recognition: a literature survey. ACM Comput Surv CSUR 35(4):399458

10. Tripathi S, Sharma V, Sharma S (2011) Face detection using combined skin color detector and template matching method. Int $\mathbf{J}$ Comput Appl 26(7):5-8

11. Craw I, Tock D, Bennett A (1992) Finding face features. Proc Second European Conf. Computer Vision, pp 92-96

12. Lanitis A, Taylor CJ, Cootes TF (1995) An automatic face identification system using flexible appearance models. Image Vis Comput 13(5):393-401

13. Vezhnevets V, Sazonov V, Andreeva A (2003) A survey on pixel-based skin color detection techniques. In: Proc. Graphicon, Moscow, Russia. vol 3, pp 85-92

14. Bhatia A, Srivastava S, Agarwal A (2010) Face detection using fuzzy logic and skin color segmentation in images. In: Emerging Trends in Engineering and Technology (ICETET), 2010 3rd International Conference, IEEE, pp 225-228

15. Leung TK, Burl MC, Perona P (1995) Finding faces in cluttered scenes using random labeled graph matching. In: Proc Fifth IEEE Int Conf Computer Vision, pp 637-644

16. Dai Y, Nakano Y (1996) Face-texture model based on SGLD and its application in face detection in a color scene. Pattern Recognit 29(6):1007-1017

17. Yang J, Waibel A (1996) A real-time face tracker. In: Proc. Third Workshop Applications of Computer Vision, pp 142-147

18. Kjeldsen R, Kender J (1996) Finding skin in color images. In: Proc Second Int Conf Automatic Face and Gesture Recognition, pp 312-317

19. Yang G, Huang TS (1994) Human face detection in a complex background. Pattern Recognit 27(1):53-63

20. Yang G, Huang TS (1994) Human face detection in complex background. Pattern Recognit 27(1):53-63

21. Rowley H, Baluja S, Kanade T et al (1998) Neural network-based face detection. Pattern Anal Mach Intell IEEE Trans 20(1):23-38

22. Turk M, Pentland A (1991) Eigenfaces for recognition. J Cogn Neurosci 3(1):71-86

23. Sung K-K, Poggio T (1998) Example-based learning for viewbased human face detection. IEEE Trans Pattern Anal Mach Intell 20(1):39-51

24. Rowley H, Baluja S, Kanade T (1998) Neural network-based face detection. IEEE Trans Pattern Anal Mach Intell 20(1):23-38

25. Osuna E, Freund R, Girosi F (1997) Training support vector machines: an application to face detection. In: Proc IEEE Conf Computer Vision and Pattern Recognition, pp 130-136 
26. Chang CF. Light compensation

27. Terrillon J-C, Shirazi MN, Fukamachi H, Akamatsu S (2000) Comparative performance of different skin chrominance models and chrominance spaces for the automatic detection of human faces in color images. In: Automatic Face and Gesture Recognition, Proceedings. Fourth IEEE International Conference IEEE, pp 54-61

28. Bergasa LM, Mazo M, Gardel A, Sotelo M, Boquete L (2000) Unsupervised and adaptive Gaussian skin-color model. Image Vis Comput 18(12):987-1003

29. Jones MJ, Rehg JM (2002) Statistical color models with application to skin detection. Int J Comput Vis 46(1):81-96

30. Wang Y-Q (2014) An analysis of the Viola-Jones face detection algorithm. Image Process On Line 4:128-148
31. Hsu R-L, Abdel-Mottaleb M, Jain AK (2002) Face detection in color images. Pattern Anal Mach Intell IEEE Trans 24(5):696-706

32. Yadav S, Nain N (2015) Fast face detection based on skin segmentation and facial features. 11th International Conference on Signal-Image Technology Internet-Based Systems (SITIS), pp 663-668

33. Milborrow S, Morkel J, Nicolls F (2010) The MUCT landmarked face database. Pattern Recognition Association of South Africa. http://www.milbo.org/muct, https://facedetection. com/datasets/. Accessed 15 Aug 2015

34. http://fei.edu.br/ cet/facedatabase.html. Accessed 12 Jan 2016

35. Tarrés F, Rama A. GTAV Face Database. http://gps-tsc.upc.es/ GTAV/ResearchAreas/UPCFaceDatabase/GTAVFaceDatabase. htm. Accessed 12 Jan 2016 